ORDOÑEZ VÁSQUEZ, Adriana; SUAREZOBANDO, Fernando. Defectos del tubo neural y del ácido fólico: recorrido histórico de una intervención preventiva altamente efectiva. História, Ciências,

Saúde - Manguinhos, Rio de Janeiro, v.22, n.4, out.-dez. 2015, p.1157-1172.

\title{
Defectos del tubo neural y del ácido fólico: recorrido histórico de una intervención preventiva altamente efectiva
}

\section{Neural tube defects and folic acid: a historical overview of a highly successful preventive intervention}

Adriana Ordoñez Vásquez

Profesor, Instituto de Genética Humana/Facultad de Medicina/ Pontificia Universidad Javeriana. Carrera 7, 40-62, Edificio 32

110311 - Bogotá D.C. - Cundinamarca - Colombia aordonez@javeriana.edu.co

\section{Fernando Suarez-Obando}

Profesor, Instituto de Genética Humana/Facultad de Medicina/ Pontificia Universidad Javeriana. Carrera 7, 40-62, Edificio 32 110311 - Bogotá D.C. - Cundinamarca - Colombia fernando.suarez@javeriana.edu.co

\section{Resumen}

Este artículo describe de forma comprehensiva parte del devenir histórico que han tenido, el conocimiento médico de los defectos del tubo neural (DTN) y el descubrimiento de la vitamina $\mathrm{B} 9$ o ácido fólico, así como algunos de los acontecimientos investigativos relevantes que a través de varios siglos definieron las relaciones entre la comprensión de la embriología del sistema nervioso central, el descubrimiento de la vitamina, la correlación del ácido fólico con la proliferación celular y finalmente el desarrollo de medidas preventivas de este tipo de defectos. Se pretende, a través de esta narrativa, exponer los conceptos históricamente relevantes que sustentan las acciones de índole clínico y de impacto poblacional que previenen los DTN a través del consumo preconcepcional de ácido fólico.

Palabras clave: ácido fólico; tubo neural; defectos del tubo neural; vitaminas; medicina preventiva.

\section{Abstract}

This article gives a broad overview of part of the historical evolution of medical knowledge about neural tube defects (NTD) and the discovery of vitamin B9 or folic acid, as well as some relevant research events that, over the course of several centuries, defined the relationships between the understanding of central nervous system embryology, the discovery of the vitamin, the correlation between folic acid and cell proliferation and lastly the development of preventive measures for this type of defects. This narrative allows us to examine historically relevant concepts underlying clinical actions with a populational impact that prevent NTDs via folic acid consumption prior to conception.

Keywords: folic acid; neural tube; neural tube defects; vitamins; preventive medicine. 
$\mathrm{L}$ os defectos del tubo neural (DTN) han sido objeto de asombro y estudio desde los orígenes de la teratología. El largo devenir de su comprensión etiológica es el reflejo de la historia de la medicina: un recorrido, originado en el ideario sobrenatural, cuyo desarrollo deviene en la medicina preventiva. Desde los registros tempranos de los naturalistas hasta el impacto epidemiológico de la suplementación de alimentos de consumo masivo, la relación entre los DTN y el ácido fólico es una historia que integra la embriología clásica, la epidemiologia y la salud pública con la bioquímica, la biología molecular y la genética clínica, en la comprensión de un hecho clínico de gran relevancia. El presente ensayo recorre los principales hechos históricos que han definido la comprensión de una de las malformaciones congénitas más frecuentes, la cual ha venido decreciendo en su prevalencia gracias a una efectiva intervención nutricional de beneficio para la salud materno fetal: el consumo preconcepcional de ácido fólico (López-Camelo, Castilla, Orioli, 2010; Rofail et al., 2012).

\section{Descripciones tempranas de los defectos del tubo neural}

El zoólogo y naturalista francés Etienne Geoffrey Saint-Hilaire reportó en 1826 el caso de anencefalia más antiguo del que se tenga registro. Saint-Hilaire describió con detalle una momia anencefálica perteneciente a una colección de piezas arqueológicas recolectadas en Tebas y Hermópolis durante las expediciones napoleónicas (1798-1801) (Goh et al., 2006). Esta momia egipcia, de alrededor de tres mil años de antigüedad estaba acompañada de un amuleto tallado en piedra representando a un babuino. En el antiguo Egipto, el Dios Babi o Baba era representado por el babuino Hamadryas (Papio hamadryas), papión sagrado o babuino sagrado egipcio. Es posible que el feto anencefálico fuera reconocido, por similitud fenotípica, con el primate Hamaydras y debido a su identificación con la deidad, fuera conservado junto con el amuleto (Saint-Hilaire, 1837; Charon, 2005) (Figura 1). Este caso de DTN no fue el único descrito por Saint-Hilaire, quien en su Histoire générale et particulière des anomalies de l'organisation chez l'homme et les animaux (Historia general y particular de la organización de las anomalías en el hombre y los animales) describió, además de la momia, casos de encefalocele y otros defectos del tubo neural. Sin embargo, el de la momia egipcia se constituye en el caso de anencefalia más antiguo del que se tenga registro (Charon, 2004).

Antes de la descripción de Saint-Hilaire, diversas representaciones precientíficas de posibles casos de DTN fueron relatadas en obras de corte recopilatorio en los siglos XVI y XVII, tales como el Prodigiorum ac ostentorum chronicon (Cronología de prodigios y portentos) de Lykosthenes (Charon, 2004; Obladen, 2011) o en la obra Des monstres et prodiges (Monstruos y prodigios) del cirujano y anatomista Ambroise Paré (Bates, 2005; Paré, 1986). En estas obras las descripciones correspondían a casos cuya descripción los ubicaba en eventos de corte anecdótico, mítico o fabuloso, sin que se diera algún tipo de planteamiento anatómico o fisiológico o una relación con anormalidades fetales específicas. Su principal valor radica en que son los registros más tempranos de patologías que, aunque relativamente infrecuentes, han impresionado tanto a médicos como cronistas a lo largo de la historia. La relación fisiopatológica entre los DTN y el desarrollo fetal se inició junto con el desarrollo de las bases de la embriología moderna. 
Los estudios científicos de la embriología del tubo neural se iniciaron alrededor de 1672 con los análisis microscópicos de embriones de pollo, llevados a cabo por Malpighi quien perfiló con detalle el prosencéfalo, el mesencéfalo y el metencéfalo (Motta, 1989). Wolff continuó trabajando en embriones de pollo, explicando con claridad la totalidad del desarrollo del tubo neural. En sus disertaciones Theoria generationis de 1759 y De formatione intestinorum, publicadas entre 1768 y 1769, Wolff formuló la noción del desarrollo embrionario de acuerdo a las capas germinales (Eisner, Bloom, 2002). Posteriormente Tiedemann realizó estudios histopatológicos en embriones humanos, detallando el proceso de formación del tubo, publicando sus resultados en 1816 en su libro clásico de embriología, sobre la anatomía del cerebro fetal (Aulie, 1961). Las descripciones detalladas de Tiedemann le permitieron a Kölliker demostrar en 1861 la base celular del cierre del tubo (Horder, 2001; Handrigan, 2003) y a His proponer que el cierre era análogo al pandeo laminar, concepto que se fundamenta en que ciertas fuerzas mecánicas actúan desde los flancos del embrión hacia la línea media, produciendo el cierre del tubo (Gordon, 2006); ambas aproximaciones, la celular y la mecánica, cimentaron el desarrollo conceptual de las fuerzas morfomecánicas, noción fundamental en el entendimiento actual del desarrollo embrionario (Taber, 2009).

Las primeras explicaciones científicas de los DTN, basadas en el estudio directo de fetos anencefálicos, fueron planteadas por Marcot y Morgagni. Marcot en 1716 sugirió que la anencefalia era el resultado de una severa hidrocefalia que comprimía la sustancia cerebral (citado por Moscoso, 2001). Por su parte, Morgagni propuso una explicación similar en 1769, señalando además que la mayoría de fetos afectados por anencefalia eran del sexo femenino (Charon, 2004; Zani, Cozzi, 2008), observación que ha sido sugerida en estudios epidemiológicos modernos: los fetos femeninos son más propensos a los defectos de neurulación, mientras que los fetos masculinos son más propensos a los defectos de canalización (Seller, 1986, 1995).

La recurrencia familiar de DTN sindrómicos fue descrita por primera vez por Meckel, al reportar en 1822 la asociación de encefalocele, polidactilia y riñón poliquístico en casos familiares recurrentes, sugiriendo de esta forma la presencia de un componente hereditario ${ }^{1}$ (Clark, 1969). Sin embargo, en 1844, Meissner atribuyó la recurrencia de casos de DNT a factores nutricionales, señalando que la desnutrición y la caquexia eran los factores desencadenantes de la espina bífida recurrente (Meissner, 2011). A pesar de la observación de Meissner, la relación de los DTN y el componente nutricional no se tuvo en cuenta hasta pasada la primera mitad del siglo XX.

A pesar de los adelantos científicos que se dieron desde el siglo XVII sobre la comprensión de la embriología del tubo neural, las explicaciones sobre los DTN seguían basándose en las elucubraciones clásicas de la teratología temprana: la monstruosidad era el resultado de una impronta fetal originada en la imaginación materna durante la concepción y el embarazo; si la madre veía o imaginaba a un primate no humano, el feto sufriría las consecuencias fenotípicas de la impresión trasmitida por la madre, y el feto sería una remembranza de lo "vivido" por su madre (Van Heiningen, 2011); es así como en Europa diversos casos de anencefalia fueron comparados por similitud con un chimpancé y relacionados con las experiencias traumáticas maternas (Obladen, 2011). En esta ocasión, no por relación divina (como en el caso de Babi) sino por la imaginación vívida de la madre durante un supuesto período crítico de formación. 


\section{Manejo nutricional de las enfermedades: el descubrimiento de las vitaminas}

La historia del descubrimiento de los micronutrientes está ligada al desarrollo del primer experimento clínico, el cual fue llevado a cabo por el cirujano James Lind. Lind tenía como propósito tratar una enfermedad específica: el escorbuto. En 1747, reclutó a 12 marinos afectados por la enfermedad; a cinco parejas de marinos les prescribió vinagre, mostaza y otros potenciales remedios, todos los marinos tratados de esta forma permanecieron afectados por el escorbuto. La pareja restante recibió naranjas y limones; las frutas cítricas mejoraron a los pacientes y la enfermedad remitió por completo (Baron, 2009). James Lind atribuyó equivocadamente el éxito de los cítricos a su supuesto poder digestivo (Bartholomew, 2002); el verdadero componente curativo, el ácido ascórbico o vitamina C, siguió siendo desconocido hasta el siglo XX. Los hallazgos de James Lind fueron ignorados por más de 40 años antes de convertir el consumo de limones en una recomendación rutinaria para los marinos ingleses durante sus travesías (Baron, 2009).

Aunque la experiencia clínica de James Lind no condujo directamente al descubrimiento de la vitamina $\mathrm{C}$, sus recomendaciones terapéuticas fueron confirmadas por el descubridor de la vitamina C, Albert Szent-György en 1927 (Magiorkinis et al., 2011). Hasta bien entrado el siglo XIX, los estudios sobre los componentes básicos de la nutrición concluían casi de forma dogmática que los requerimientos dietéticos se basaban únicamente en calorías, proteínas y la necesidad de ciertos minerales (Nichols, 2001), sin embargo fueron los trabajos pioneros de Lunin los que dieron origen al estudio de otros componentes nutricionales fundamentales para la vida. En 1881, Lunin experimentó con ratones alimentándolos con dietas basadas exclusivamente en proteína, grasa y sal. Los ratones morían después de 16 a 36 días, no obstante al añadir pequeñas cantidades de leche, los ratones sobrevivían meses. Lunin concluyó que en la leche debía existir algún elemento esencial para la vida, sin embargo en la época se asumió que ese componente esencial correspondía realmente a un suplemento calórico adicional (Wolf, Carpenter, 1997). Aunque otros autores replicaron los hallazgos de Lunin, sólo los estudios de Eijkman condujeron directamente hacia el descubrimiento de las vitaminas.

Eijkman llevó a cabo una serie de experimentos en pollos, alternando su dieta de arroz con y sin cascarilla y relacionando la aparición de polineuritis en las aves con el consumo de arroz sin cascarilla; Eijkman describió sus hallazgos en una serie de informes fechados entre 1889 y 1900. Los trabajos de Eijkman condujeron al tratamiento del beri beri con dietas que contenían cereales completos, como por ejemplo el arroz con cascarilla. Eijkman² concluyó que además de la cascarilla, la cocción y el agua tendrían que ver con la neutralización de una toxina y un posible proceso infeccioso que desencadenaba la enfermedad (Carpenter, Sutherland, 1995). Posteriormente se estableció que el beri beri era secundario a la deficiencia de tiamina, la cual fue cristalizada en 1926 por Jansen and Donath (Lanska, 2010a).

Pekelharing en 1905 y Hopkins en 1912 utilizaron técnicas experimentales similares a las de Eijkman, usando dietas purificadas para ratones y llegando a la misma conclusión que Lunin: la existencia de factores nutricionales eran accesorios esenciales para el desarrollo y mantenimiento de la salud (Lanska, 2010b; Braun, 2011). 
Estos factores accesorios fueron por primera vez llamados de vitaminas por Funk en 1911. Funk aisló un concentrado de cascarilla de arroz que curaba la polineuritis en palomas, sus análisis indicaron que el concentrado curativo probablemente contenía una amina, y como esta sustancia parecía ser necesaria para la vida, Funk la denominó "vita-amina"; aunque las vitaminas no son aminas, el nombre permaneció como denominador común de estos micronutrientes (Rosenfeld, 1997; Piro et al., 2010). Durante la primera mitad del siglo XX se aislaron la mayoría de vitaminas lipo- e hidrosolubles, siguiendo en principio, los mismos métodos de Lunin y Eijkman: correlacionando dietas específicas con manifestaciones clínicas, tal fue el caso del ácido fólico y la anemia megaloblástica del embarazo.

En 1928, Wills estableció que la anemia megaloblástica de ratas albinas producida por una dieta deficiente en vitamina $\mathrm{B}$, era prevenida con la adición de extracto de levadura. En 1935, Day indujo experimentalmente anemia y leucopenia en monos Rhesus, luego de alimentarlos con dietas carentes de vitamina B (Day, Langston, Shukers, 1935). En un experimento similar, Wills reprodujo la anemia macrocítica nutricional en la misma especie de monos Rhesus, corrigiéndola con el mismo preparado de extracto de levadura utilizado en la ratas albinas, demostrando así por primera vez, la cura de esta enfermedad en primates empleando exclusivamente un manejo nutricional (Wills, Stewart, 1935).

La anemia megaloblástica del embarazo fue corregida utilizando el mismo extracto en mujeres embarazadas con severas deficiencias nutricionales (Wills, 1991), inclusive, la anemia megaloblástica hipercrómica secundaria a la enfermedad celiaca y a la esteatorrea idiopática, también fue corregida con el mismo extracto de levadura; similares resultados curativos se obtuvieron utilizando alternativamente el extracto de levadura o el extracto crudo de hígado, sugiriendo la existencia de un factor nutricional correctivo con el mismo efecto clínico que la levadura, pero localizado en el hígado.

Los experimentos clínicos llevados a cabo en Bombay por la Dra. Wills utilizaron como fuente de levadura a la Marmita ${ }^{3}$ y el factor correctivo de la anemia se conoció como el factor de Wills (Hultquist et al., 1947; Hoffbrand, Weir, 2001).

El factor de Wills fue posteriormente denominado folato, luego de ser aislado por Mitchell de las hojas de la espinaca en $1941 .{ }^{4}$ Mitchell también demostró que el folato era el factor de crecimiento de los cultivos de la bacteria Streptococo faecalis, aspecto que cobraría enorme relevancia en los futuros estudios sobre los antagonistas de crecimiento tumoral (Mitchell, Snell, Williams, 1988).

El compuesto vitamínico fue aislado en su forma cristalina (fólico) por Stokstad en 1943 (Shane, Carpenter, 1997). Stokstad determinó que el ácido fólico está compuesto por un anillo de pteridina, ácido paraaminobenzoico y acido glutámico, el ácido fólico se denominó ácido pteroilglutámico (Angier et al., 1948). El término ácido fólico se utiliza para denominar la forma oxidada del compuesto químico, la cual no está presente en la naturaleza. El término folato es utilizado para denominar los compuestos con la misma actividad vitamínica incluyendo las forma naturales y al ácido fólico propiamente dicho (Hoffbrand, Weir, 2001).

La relación definitiva entre la deficiencia de folato y la anemia megaloblástica, fue comprobada en 1962 con el dramático experimento de Víctor Herbert, quien se sometió a una dieta deficiente en folatos, monitoreando él mismo los eventos hematológicos y midiendo los niveles plasmáticos de la vitamina. Herbert estableció, al cabo de quatro meses 
de experimentación, la relación clínica entre la deficiencia vitamínica y la anemia nutricional (Herbert, 1962, 1963; Scott, 2004).

Dado que los trastornos hematológicos, como la anemia no relacionada con la carencia de hierro, se corregían con el suplemento de ácido fólico, rápidamente se comenzó a experimentar acerca de su uso en el tratamiento de otras patologías, especialmente en cáncer.

\section{Los antagonistas del ácido fólico}

En 1945 Leuchtenberger reportó la remisión de cáncer espontáneo de mama en ratones, con la inyección intravenosa de ácido fólico (Leuchtenberger et al., 1945). Este tipo de hallazgos estimuló el manejo de patologías hemato-oncológicas con el compuesto vitamínico. Sin embargo el uso del ácido fólico en trastornos como la leucemia generó una reacción totalmente opuesta: el fenómeno de aceleración del proceso leucémico en la médula ósea de niños con leucemia aguda tratados con la inyección de conjugados de ácido fólico. Este fenómeno reportado por Farber en 1947 llevó a pensar a los investigadores que se podría contrarrestar el crecimiento celular anómalo con antagonistas del ácido fólico (Farber et al., 1947).

Entre 1947 y 1948 Oleson y Seeger describieron diversos antagonistas del ácido pteroilglutámico (ácido fólico) tales como el ácido aspártico y la aminopterina. El efecto inhibitorio, específicamente de la aminopterina, se estableció de forma indirecta. De acuerdo con Oleson, la aminopterina inhibía el crecimiento in vitro del Streptococo faecalis, microorganismo que crecía en presencia del ácido fólico (Seeger et al., 1947; Oleson et al., 1948). Olenson describió además que las ratas alimentadas con aminopterina tenían medula ósea hipoplásica y que los cambios histopatológicos revertían con altos niveles de ácido fólico (Oleson et al., 1948). Luego de reportar el fenómeno de aceleración del proceso leucémico en medula ósea y de conocer el trabajo de Oleson, Farber llevó a cabo en 1948 el primer tratamiento de quimioterapia para el cáncer, utilizando aminopterina en casos avanzados de leucemia. Farber reportó la remisión parcial de la enfermedad en cinco pacientes, inaugurando el desarrollo de la quimioterapia para el manejo del cáncer (Farber, Diamond, 1948).

\section{Metabolismo del ácido fólico}

El mecanismo de acción de la aminopterina fue dado a conocer en 1950 con los trabajos de Nichol y Welch (1950) y Greenspan y colaboradores (1950). Estos trabajos pioneros establecieron los elementos esenciales para describir la totalidad de las vías metabólicas que involucran al ácido fólico.

Para finales de la década del 1950 e inicio de la década del 1960, los principales aspectos del metabolismo del ácido fólico eran conocidos (Bertino et al., 1963). Por lo tanto, cabe resaltar cuatro aspectos esenciales para comprender la relación entre el ácido fólico y los DTN. En primer lugar, el mecanismo de acción de la aminopterina que consiste en inhibir la enzima dihidrofolato reductasa (DFR), enzima que cataliza la conversión del dihidrofolato (DHF) a tetrahidrofolato (THF) (Greenspan et al., 1951). En segundo lugar, la participación directa del ácido fólico en la síntesis del ADN a través de la reacción de síntesis de los nucleótidos de timina, reacción mediada por la presencia de THF. Esta reacción fue detallada por Pastore 
en 1962, (Pastore, Friedkin, 1962; Cooper, Milton, 1964) y dos años despues verificada por Killmann (1964). En tercer lugar, al tener en cuenta el acelerado crecimiento celular durante el inicio del embarazo (desarrollo fetal y placentario) Chanarin en 1968, determinó que el estado fisiológico del embarazo incrementa los requerimientos de ácido fólico (Chanarin, Rothman, Perry, 1968). Por último Rosemberg en 1969, demostró que los folatos eran reducidos, metilados y deconjugados a 5-tetrahidrofolato (THF) durante la absorción intestinal (Rosenberg et al., 1969).

El incremento de los requerimientos de la vitamina durante el embarazo, la absorción intestinal de la vitamina y el requerimiento de ácido fólico en la síntesis de ADN, definen en gran proporción la relación entre los DTN y su suplemento periconcepcional. Asimismo, la inhibición de su metabolismo establece una clara relación entre los mecanismos teratogénicos de la aminopterina y los DTN. Sin embargo, aunque estas conexiones eran claras a nivel bioquímico y fisiológico, las relaciones clínicamente significativas entre el metabolismo del ácido fólico y la prevención de los DTN se dedujeron solamente con base en estudios epidemiológicos.

Por otra parte, el efecto inmunosupresor de los antagonistas del ácido fólico ha resultado beneficioso en el manejo de otras patologías autoinmunes como la enfermedad intestinal inflamatoria (Egan, Sandborn, 1996) e incluso ha demostrado ser efectivo en el manejo de patologías parasitarias como la malaria, cuyo efecto terapéutico probablemente se deba a una interacción entre la respuesta inmune y acción antiparasitaria directa, efectividad que parece superar los riesgos de toxicidad hepática (Nzila et al., 2010).

Debido a su efecto inmuno-modulador, el uso de los antagonistas del ácido fólico extendió su desarrollo terapéutico hacia el manejo de otras enfermedades, alcanzando especial éxito en el manejo de enfermedades autoinmunes como la psoriasis, y artritis reumatoide (Kersley, 1968); de igual forma, su acción inhibitoria del índice mitótico lo hizo un elemento terapéutico efectivo en el manejo del embarazo ectópico temprano (St. Clair et al., 1969) y del coriocarcinoma (Min, 1971).

El Metrotexate continúa siendo un medicamento de primera línea en el manejo de estas enfermedades.

\section{El ácido fólico y los DTN}

En 1964, la doctora Elizabeth Hibbard fue la primera en establecer que la deficiencia del folato podría jugar un papel relevante en el desarrollo de diversas patologías del embarazo (Hibbard, 1964). Hibbard observó que la deficiencia de folato durante el embarazo era secundaria al suplemento nutricional inadecuado y que las mujeres desnutridas tenían mayor riesgo de tener complicaciones del embarazo tales como: abrupción de placenta, aborto recurrente, bajo peso al nacer, malformaciones congénitas e incremento de la mortalidad perinatal (Scholl, Johnson, 2000), argumento similar al de Meissner en 1844. Hibbard y Smithells (1965) propusieron que la ocurrencia familiar de malformaciones del sistema nervioso central estaba relacionada, en algunos casos, con defectos genéticos relacionados con el metabolismo del ácido fólico, argumento que antecedió en décadas la relación de algunos 
polimorfismos enzimáticos de la metilen-tetrahidrofolato reductasa (MTHFR) como factor de riesgo para el desarrollo de DTN en algunas poblaciones (Johnson et al., 1999).

En 1976, Smithells llevó a cabo un estudio observacional de corte trasversal en el cual seis mujeres de un grupo total de 900 dieron a luz niños con DTN, las seis mujeres tenían bajos niveles de riboflavina, vitamina $\mathrm{C}$, folato sérico y folato eritrocitario. Las diferencias entre los niveles de folato eritrocitario y vitamina $\mathrm{C}$ en comparación con los niveles del resto del grupo, eran estadísticamente significativas (Smithells et al., 1976).

En 1980 Smithells publicó los resultados de la primera intervención destinada a prevenir la recurrencia de DTN con la administración periconcepcional de vitaminas. En el estudio observacional, se reclutaron mujeres que habían tenido anteriormente hijos con algún tipo de DTN. En el primer informe del estudio, se reportó que 1 de 178 recién nacidos $(0,56 \%)$, de madres que habían recibido el suplemento vitamínico, presentó DTN en comparación con 13 de 260 (5\%) recién nacidos de madres que no recibieron el suplemento vitamínico (Smithells et al., 1980). El suplemento vitamínico consistía en 0,12mg de ácido fólico, vitaminas C, B1, B2, B6 vitamina A, D y nicotinamida. La intervención de Smithells sugería un riesgo mayor de padecer DTN en mujeres sin el suplemento vitamínico. Posteriores reportes en 1983 y 1989 confirmaron la efectividad del suplemento que contenía ácido fólico (Smithells et al., 1983, 1989).

En 1981, Laurence y colaboradores llevaron a cabo el primer experimento clínico (aleatorizado doble ciego) diseñado para prevenir la recurrencia de DTN. En el experimento clínico (Laurence et al., 1981) 44 mujeres recibieron un suplemento vitamínico de ácido fólico de $2 \mathrm{mg}$ dos veces al día y 51 mujeres recibieron placebo. Las mujeres aceptaron iniciar el consumo del suplemento o el placebo desde el momento en que ellas suspendieran las medidas anticonceptivas y continuar su consumo durante las primeras semanas del embarazo. Entre las mujeres que recibieron el tratamiento y cumplieron con el régimen no se presentó ninguna recurrencia de DTN; dos mujeres del grupo de tratamiento no cumplieron con la dosificación y tuvieron recurrencia de DTN. En el grupo control (placebo) se presentaron seis recurrencias $(p=0,04)$. Los niveles de folato eritrocitario entre el grupo de tratamiento y el grupo control también tuvieron una diferencia significativa $(\mathrm{p}<0,001)$.

Laurence fue motivado a llevar a cabo el experimento clínico gracias a las conclusiones de los estudios de Hibbard y Smithells; su decisión de utilizar únicamente ácido fólico como intervención clínica y no como un multivitamínico se basó en el reporte de Thiersch (1952), en el cual se describían casos de anencefalia luego del uso de la aminopterina como agente abortivo. Es así como Laurence al reconocer el efecto específico de la aminopterina sobre la DFR, aclaró los factores de confusión generados en el estudio de Smithells de 1980, en el cual se utilizaron multivitamínicos que incluían el ácido fólico.

\section{El gran experimento clínico: The Medical Research Council (MRC)}

El diagnóstico prenatal de espina bífida y anencefalia, basado en las concentraciones séricas de alfafetoproteína (AFP), fue reportado por Wald y colaboradores en 1977. La medición de AFP fue presentada como un método efectivo para seleccionar mujeres que requerían ultrasonido detallado y amniocentesis (Wald et al., 1977). El mismo Wald junto 
con Polani expresaron dudas sobre el uso de multivitamínicos como medida preventiva de los DTN, indicando diferencias sistemáticas entre los grupos comparados por los estudios de Smithells, señalando además que el uso de vitamina A en los multivitamínicos para la mujer embarazada era un factor teratogénico que se debía considerar con mayor cautela. Aunque Smithells publicó en 1985 una carta en la que aclaraba las dudas expresadas por Wald y Polani (Smithells et al., 1985), la controversia apuntaba a la necesidad de un experimento clínico que pudiese esclarecer definitivamente las dudas. Es así como en 1984, Wald, Polani y colaboradores propusieron el desarrollo de un experimento clínico aleatorizado con placebo con el objetivo de establecer el verdadero papel del ácido fólico en la prevención de los DTN (Wald et al., 1984).

El experimento clínico de Wald y Polani fue llevado a cabo por la institución británica Medical Research Council, entidad que en 1983 inició el reclutamiento de mujeres con alto riesgo de padecer un embarazo afectado por DTN debido a antecedentes de embarazos previos afectados por el mismo tipo de malformación (Beardsley, 1983; Abel, 1984).

El estudio del MRC se extendió hasta 1991, reclutando a un total de 1.817 mujeres en 33 centros de siete países (17 centros en el Reino Unido). Cada una de las participantes fue asignada aleatoriamente a uno de los cuatro grupos de suplementación vitamínica previa al embarazo (grupos A, B, C y D). El grupo A recibió 4mg de ácido fólico; el grupo B recibió un complejo multivitamínico además de $4 \mathrm{mg}$ de ácido fólico; el grupo C no recibió ni el multivitamínico ni el ácido fólico, mientras que el grupo D recibió el multivitamínico sin el ácido fólico. Todas las cápsulas (incluídas las del grupo C) contenían suplementos de sulfato ferroso (120mg) y calcio $(240 \mathrm{mg})$. La medida de desenlace fue la recurrencia de DTN comparando los grupos A y B con los grupos C y D.

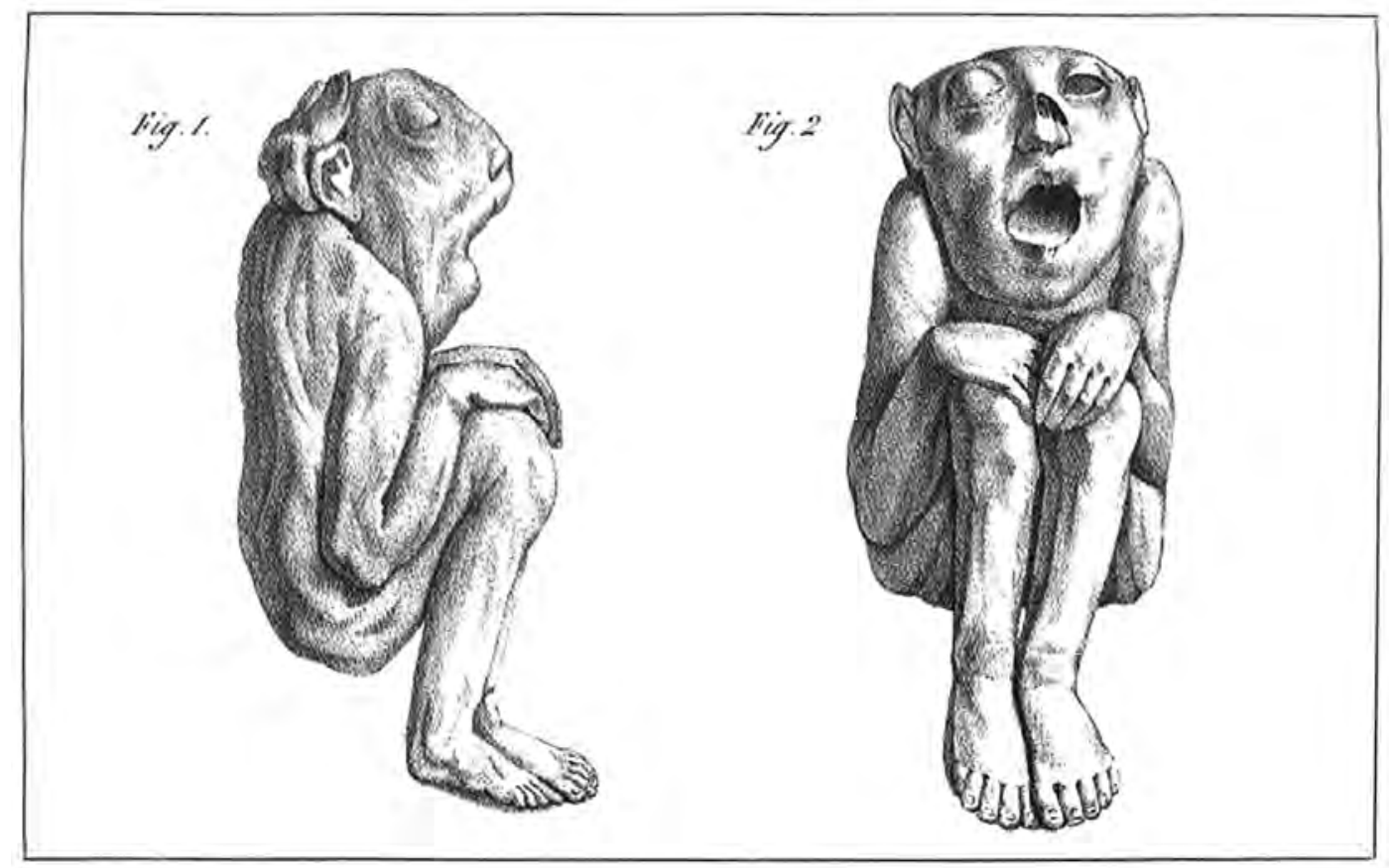

Figura 1: Momias anencefálicas (Saint-Hilaire, 1837, plancha 9) 
Se llegaron a las siguientes conclusiones utilizando la información disponible de 1.195 embarazos: recurrencia de DTN en seis de 593 recién nacidos o fetos de mujeres que recibieron ácido fólico (1\%). En el grupo A: dos de 298 y en el grupo B: cuatro de 295. En los grupos que no recibieron ácido fólico la recurrencia de DTN fue de 21 de 602 recién nacidos o fetos (3,5\%): 13 casos de 300 en el grupo C y ocho casos de 302 en el grupo D. En conclusión, el consumo de ácido fólico antes del embarazo se asoció a una reducción del 71\% del riesgo de recurrencia de DTN (MRC..., 1991; Centers..., 1991).

Es así como los resultados fueron consistentes con los estudios de Laurence, Smithellis y con los estudios publicados posteriormente por Czeizel (Czeizel, Dudas, 1992).

\section{Consideraciones finales}

El impacto de la propuesta original de Smithellis, y su confirmación por diversos experimentos clínicos, se ve reflejado en las múltiples recomendaciones basadas en la evidencia que indican la necesidad de consumir ácido fólico antes del embarazo con el propósito de prevenir los DTN (Wolff et al., 2009; De-Regil et al., 2010). Sin embargo, la mayoría de embarazos son eventos no planeados (Trussell, 2007) y existe poco conocimiento sobre la importancia del consumo de ácido fólico aun en zonas de alta prevalencia de los DTN (Canfield et al., 2002; Ren et al., 2006), de tal forma que la importancia del consumo de la vitamina tiene que ser promocionado a través de campañas dirigidas a mujeres en edad fértil o administrado a la población a través de la fortificación de alimentos de consumo masivo.

La fortificación de alimentos ha demostrado ser una medida útil en la prevención de los DTN, por ejemplo en los Estados Unidos luego de la fortificación obligatoria de granos que

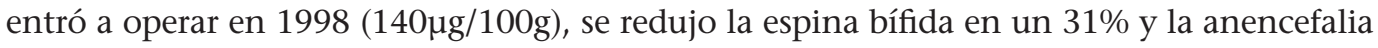
en un 16\% (Williams et al., 2002), mientras que en Chile con la fortificación de la harina $(220 \mu \mathrm{g} / \mathrm{kg}$ ) se redujo la prevalencia de DTN en un 46\% (López-Camelo, Castilla, Orioli, 2005); sin embargo la reducción ha sido menor a lo esperado, lo cual puede ser atribuido a que se requieren mayores concentraciones en la fortificación (Johnston Jr., 2008) o a que las mujeres no acceden a los alimentos fortificados (Tedstone et al., 2008). Una alternativa de prevención, además de la fortificación de los alimentos, es la suplementación nutricional promocionada a través de campañas dirigidas tanto a mujeres como a médicos que prescriben la vitamina antes del embarazo (Bower et al., 2005), medida que ha proporcionado resultados prometedores siempre y cuando las campañas continúen y permanezcan en el tiempo (Rofail et al., 2012).

La relación entre ácido fólico y el desarrollo de los DTN ha sido mejor detallada con la comprensión de la relación entre los polimorfismos de las enzimas del metabolismo de la vitamina y el desarrollo embrionario. Por ejemplo, se ha sugerido que los polimorfismos del gen de la Metilen Tetrahidro Folato Reductasa (MTHFR), maternos y fetales, podrían tener un papel en el riesgo incrementado de desarrollo de DTN (Etheredge et al., 2012), sin embargo los estudios no han sido conclusivos y los resultados varían entre poblaciones, así como el riesgo atribuible a diversos genes involucrados en el metabolismo de la vitamina (Pangilinan et al., 2012; Wang et al., 2012).

Otros factores de riesgo independientemente relacionados con el desarrollo de los DTN son los bajos niveles séricos de vitamina B12, niveles elevados de homocisteina (Zhang et 
al., 2009), y obesidad (Tinker et al., 2012), demostrando la naturaleza multifactorial de los DTN que además de las deficiencias de ácido fólico, incluye factores ambientales como la exposición a micotoxinas como la fumosina (Missmer et al., 2006).

El recorrido que ha tenido el entendimiento de las relaciones entre la nutrición y los DTN no concluye en la intención de prevenir las malformaciones congénitas con el consumo preconcepcional del micronutriente, sino que se extiende a mantener el consumo entre las mujeres en edad fértil - fortificación, campañas de promoción y prevención, anticonceptivos con ácido fólico (Lassi, Bhutta, 2012) - y puede involucrar la prevención de otras malformaciones como el labio y el paladar hendido (Kelly et al., 2012) y patologías oncológicas como el cáncer de colon (Sie et al., 2011), además, existen otros aspectos de la fortificación que deben aclararse, tales como la posible relación entre el consumo de ácido fólico durante el embarazo y el desarrollo de alergias respiratorias, incremento en el riesgo de procesos de tumorogénesis diferentes al cáncer de colon, embarazos gemelares y resistencia a la insulina (Burdge, Lillycrop, 2012). Sin duda el consumo preconcepcional de ácido fólico ha sido una medida eficaz y de gran impacto en la salud pública, impacto que ha hecho posible la disminución en la prevalencia de malformaciones severas, las cuales, se espera, queden atrás en el tiempo, únicamente como registros históricos.

\section{APÉNDICE: GLOSARIO}

Alfa-fetoproteína: proteína codificada por el gen AFP (4q25). Es la proteína fetal más abundante del embrión humano. Se cree que es el equivalente fetal de la albúmina del adulto. Sus niveles se encuentran elevados en el líquido amniótico o sangre materna cuando el feto presenta defectos del tubo neural. Sus niveles también son un marcador de onfalocele, trisomía 21, o tumores del saco vitelino. En el adulto es un marcador de tumores del saco vitelino, y carcinoma hepatocelular, entre otros.

Aminopterina: antifolato que bloquea la actividad de la síntesis de tetrahidrofolato, es un análogo del ácido fólico y se deriva de la pterina.

Amniocentesis: procedimiento en el cual se extrae el líquido amniótico. En el líquido hay células fetales susceptibles de análisis moleculares y citogenéticas.

Anemia megaloblástica: carencia de glóbulos rojos, en la cual los eritrocitos tienen gran tamaño. Resulta de la carencia de vitamina B12, de ácido fólico o de ambas.

Defectos del tubo neural: anomalías congénitas del sistema nervioso central y de la médula espinal, resultantes de la ausencia o del cierre incompleto del tubo neural en desarrollo.

Encefalocele: defecto del tubo neural en el tejido cerebral; las meninges protruyen a través de un defecto en la bóveda del cráneo.

Eritrocito: elementos formes de la sangre que contienen hemoglobina y trasportan oxígeno hacia los tejidos.

Escorbuto: avitaminosis producida por la carencia de vitamina C.

Espina bífida: defecto del tubo neural en el cual uno o varios arcos vertebrales no se han fusionado, puede estar acompañado de meningocele o de mielomeningocele.

Hidrocefalia: acumulación anormal de líquido en el cerebro, se asocia, en la población pediátrica, a un incremento en el perímetro cefálico.

Leucemia: tipo de enfermedad oncológica que se caracteriza por el incremento en el número de células blancas de la sangre o leucocitos.

Leucopenia: disminución del número de células blancas (leucocitos) en la sangre.

Meningocele: protrusión de las meninges a través de un defecto de fusión de los arcos vertebrales. 
Mielomeningocele: protrusión de las meninges y de médula espinal a través de un defecto de fusión de los arcos vertebrales.

Polidactilia: dedo supernumerario de las manos o de los pies.

Teratógeno: agente químico o físico con la capacidad de alterar el desarrollo embriológico y causar una anomalía congénita.

\section{NOTAS}

${ }^{1}$ Esta descripción sindrómica realmente corresponde al síndrome de Meckel-Gruber, síndrome de Gruber o disencefalia esplancnoquística, síndrome de herencia autosómica recesiva, causado por mutaciones en el gen MKS1.

${ }^{2}$ Hopkins y Eijkman fueron galardonados con el premio Nobel de medicina y fisiología de 1929 por el descubrimiento de las vitaminas.

${ }^{3}$ Marmita: suplemento nutricional hecho de concentrado de Saccharomyces cerevisiae. La Marmite Food Extract Company comercializaba en el Reino Unido el concentrado. En la etiqueta del producto se presentaba el recipiente metálico de cocina, la marmita, de donde tomó su nombre el suplemento utilizado por Wills.

${ }^{4}$ Mitchell sugirió el nombre folato, del latín folium (hoja).

\section{REFERENCIAS}

ABEL, Josef.

Neural tube defects: British vitamin trial starts slowly. Nature, v.309, n.5970, p.661. 1984.

ANGIER, Robert et al.

Synthesis of pteroylglutamic acid. Journal of the American Chemical Society, v.70, n.1, p.25. 1948.

AULIE, Richard.

Caspar Friedrich Wolff and his "Theoria generationis", 1759. Journal of the History of Medicine and Allied Sciences, v.16, n.2, p.124-144. 1961.

BARON, Jeremy Hugh.

Sailors' scurvy before and after James Lind-a reassessment. Nutrition Reviews, v.67, n.6, p.315332. 2009.

BARTHOLOMEW, Mary.

James Lind's Treatise of the scurvy (1753).

Postgraduate Medical Journal, v.78, n.925, p.695696. 2002.

BATES, Alan W.

Emblematic monsters: unnatural conceptions and deformed births in early modern Europe. Amsterdam: Rodopi. 2005.

BEARDSLEY, Tausha.

Spina bifida: MRC folate trials to start at last. Nature, v.303, n.5919, p.647. 1983.

BERTINO, Joseph et al.

Studies on normal and leukemic leukocytes. IV. Tetrahydrofolate-dependent enzyme systems and dihydrofolic reductase. Journal of Clinical Investigation, v.42, n.12, p.1899-1907. 1963.
BOWER, Carol et al.

Promotion of folate for the prevention of neural tube defects: who benefits? Paediatric and perinatal epidemiology, v.19, n.6, p.435-444. 2005.

BRAUN, Robyn.

Accessory food factors: understanding the catalytic function. Journal of the History of Biology, v.44, n.3, p.483-504. 2011.

BURDGE, Graham C.; LILLYCROP, Karen A. Folic acid supplementation in pregnancy: are there devils in the detail? The British Journal of Nutrition, v.108, n.11, p.1924-1930. 2012.

CANFIELD, Mark A. et al.

Folic acid awareness and use among women with a history of a neural tube defect pregnancy: Texas, 2000-2001. MMWR Recommendations and reports, Centers for Disease Control, v.51, n.RR13, p.16-19. 2002.

CARPENTER, Kenneth J.; SUTHERLAND, Barbara Eijkman's contribution to the discovery of vitamins. Journal of Nutrition, v.125, n.2, p.155163. 1995.

\section{CENTERS...}

Centers for Disease Control. Effectiveness in disease and injury prevention use of folic acid for prevention of spina bifida and other neural tube defects: 1983-1991. MMWR Weekly, v.40, n.30, p.513-516. 1991.

CHANARIN, Id; ROTHMAN, Doreen; PERRY, Janet. Folate status and requirement in pregnancy. British Medical Journal, v.2, n.5602, p.390-394. 1968. 
CHARON, Pierre.

Teratology of the neural tube: history and paleopathology. Antropo, n.10, p.83-101. 2005.

CHARON, Pierre.

Etienne Geoffrey Saint-Hilaire (1772-1844) and anencephaly: contribution of one naturalist to medical knowledge. Histoire des sciences médicales, v.38, n.3, p.365-383. 2004.

\section{CLARK, Orlo.}

The contributions of J.F. Meckel, the Younger, to the science of teratology. Journal of the History of Medicine and Allied Sciences, v.24, n.3, p.310-322. 1969.

COOPER, E.H.; MILTON, J.D.

The incorporation and degradation of pyrimidine DNA precursors by human leucocytes. British Journal of Cancer, v.18, n.4, p.701-713. 1964.

CZEIZEL, Andrew; DUDAS, Istvan.

Prevention of the first occurrence of neuraltube defects by periconceptional vitamin supplementation. The New England Journal of Medicine, v.327, n.26, p.1832-1835. 1992.

DAY, Paul L.; LANGSTON, William C.; SHUKERS, Carroll F.

Leukopenia and anemia in the monkey resulting from vitamin deficiency. Journal of Nutrition, v.9, n.5, p.637-644. 1935.

DE-REGIL, Luz Maria et al.

Effects and safety of periconceptional folate supplementation for preventing birth defects. Cochrane database of systematic reviews, v.10, n.CD007950. 2010.

EGAN, Laurence; SANDBORN, William. Methotrexate for inflammatory bowel disease: pharmacology and preliminary results. Mayo Clinic Proceedings, v.71, n.1, p.69-80. 1996.

EISNER, Brian; BLOOM, David A.

Wolff and Muller: fundamental eponyms of embryology, nephrology and urology. Journal of Urology, v.168, n.2, p.425-428. 2002.

ETHEREDGE, Analee et al.

Maternal and infant gene-folate interactions and the risk of neural tube defects. American Journal of Medical Genetics, part A, v.158a, n.10, p.24392446. 2012.

FARBER, Sídney; DIAMOND, Louis K. Temporary remissions in acute leukemia in children produced by folic acid antagonist, 4-aminopteroyl-glutamic acid. The New England Journal of Medicine, v.238, n.23, p.787-793. 1948.

FARBER, Sidney et al.

The action of pteroylglutamic conjugates on man. Science, v.106 n.2764, p.619-621. 1947.
GOH, Ingrid et al.

Prenatal multivitamin supplementation and rates of congenital anomalies: a meta-analysis. Canadian Journal of Obstetrics and Gynecology, v.28, n.8, p.680-689. 2006.

GORDON, Richard.

Mechanics in embryogenesis and embryonics: prime mover or epiphenomenon? International Journal of Developmental Biology, v.50, p.245-253. 2006.

GREENSPAN, Ezra M. et al.

Studies on the mechanism of action of chemotherapeutic agents in cancer. 5. Influence of the citrovorum factor and folic acid on the toxic manifestations of aminopterin in mice. Cancer, v.4, n.3, p.619-625. 1951.

GREENSPAN, Ezra M. et al.

Studies on the mechanism of action of chemotherapeutic agents in cancer. 2 .

Requirements for the prevention of aminopterin toxicity by folic acid in mice. Cancer, v.3, n.5, p.856-863. 1950.

HANDRIGAN, Gregory R.

Concordia discords: duality in the origin of the vertebrate tail. Journal of Anatomy, v.202, n.3, p.255-267. 2003.

HERBERT, Victor.

A palatable diet for producing experimental folate deficiency in man. American Journal of Clinical Nutrition, v.12, n.1, p.17-20. 1963.

HERBERT, Victor.

Experimental nutritional folate deficiency in man. Transactions of the Association of American Physicians, v.75, p.307-320. 1962.

HIBBARD, Bryan M.

The role of folic acid in pregnancy; with particular reference to anemia, abruption and abortion. Journal of Obstetrics and Gynecology of the British Commonwealth, v.71, p.529-542. 1964.

HIBBARD, Elizabeth; SMITHELLS, R.W.

Folic acid metabolism and human embryopathy. The Lancet, v.285, n.7398, p.1254. 1965.

HOFFBRAND, Victor; WEIR, D.G.

The history of folic acid. British Journal of Haematology, v.113, n.3, p.579-589. 2001.

HORDER, Tim.

History of developmental biology. New York: John Wiley \& Sons. 2001.

HULTQUIST, Me et al.

Folic acid supplement; synthesis of pteroylglutamic acid (liver L. Casei factor) and pteroic acid. Annals of the New York Academy of Sciences, v.48, art.5, suppl., p.i-vi. 1947. 
JOHNSON, William G. et al.

Distribution of alleles of the

methylenetetrahydrofolate reductase (MTHFR)

C677T gene polymorphism in familial spina

bifida. American Journal of Medical Genetics, v.87, n.5, p.407-412. 1999.

JOHNSTON JR., Ronald B.

Will increasing folic acid in fortified grain products further reduce neural tube defects without causing harm? Consideration of the evidence. Pediatric Research, v.63, n.1, p.2-8. 2008.

KELLY, Dervla et al.

Use of folic acid supplements and risk of cleft lip and palate in infants: a population-based cohort study. The British Journal of General Practice, v.62, n.600, p.466-472. 2012.

KERSLEY, George D.

Amethopterin (methotrexate) in connective tissue disease-psoriasis and polyarthritis. Annals of the Rheumatic Diseases The EULAR Journal, v.27, n.1, p.64-66. 1968.

KILLMANN, Sven-Aage.

Effect of deoxyuridine on incorporation of tritiated thymidine: difference between normoblasts and megaloblasts. Acta Medica Scandinavica, v.175, n.4, p.483-488. 1964.

LANSKA, Douglas J.

Chapter 29: historical aspects of the major neurological vitamin deficiency disorders: overview and fat-soluble vitamin A. Handbook of Clinical Neurology, v.95, p.435-444. 2010a.

LANSKA, Douglas J.

Chapter 30: historical aspects of the major neurological vitamin deficiency disorders: the water-soluble B vitamins. Handbook Clinical Neurology, v.95, p.445-476. 2010b.

LASSI, Zohra S.; BHUTTA, Zulfiqar.

A clinical utility of folate-containing oral contraceptives. International Journal of Women's Health, v.4, p.185-190. 2012.

LAURENCE, K. Michael et al.

Double-blind randomised controlled trial of folate treatment before conception to prevent recurrence of neural-tube defects. British Medical Journal (Clinical Research Edition), v.282, n.6275, p.1509-1511. 1981.

LEUCHTENBERGER, Rudolf et al.

The influence of "folic acid" on spontaneous breast cancers in mice. Science, v.101, n.2611, p.46. 1945.

LÓPEZ-CAMELO, Jorge S.; CASTILLA, Eduardo E.; ORIOLI, Iêda M.

Folic acid flour fortification: impact on the frequencies of 52 congenital anomaly types in three South American countries. American Journal of Medical Genetics part A, v.152a, n.10, p.24442458. 2010.

LÓPEZ-CAMELO, Jorge S.; CASTILLA, Eduardo E.; ORIOLI, Iêda M.

Reduction of birth prevalence rates of neural tube defects after folic acid fortification in Chile. American Journal of Medical Genetics part A, v.135, n.2, p.120-125. 2005.

MAGIORKINIS, Emmanuil et al.

Scurvy: past, present and future. European Journal of Internal Medicine, v.22, n.2, p.147-152. 2011.

MEISSNER, Ladurner.

Die Kinderkrankheiten Nach Den Neusten Ansichten Und Erfahrungen Zum Unterricht Fuer Praktische Aerzte Bearbeitet. v.2. Charleston: Nabu Press. 2011.

MIN, C. Li.

Trophoblastic disease: natural history, diagnosis, and treatment. Annals of Internal Medicine, v.74, n.1, p.102-112. 1971.

MISSMER, Stacey et al.

Exposure to fumonisins and the occurrence of neural tube defects along the Texas-Mexico border. Environmental health perspectives, v.114, n.2, p.237-241. 2006.

MITCHELL, Herschel K.; SNELL, Esmond E.; WILLIAMS, Roger J.

The concentration of "folic acid". Nutrition Review, v.46, n.9, p.324-325. 1988.

MOSCOSO, Javier.

Los efectos de la imaginación: medicina y ciencia y sociedad en el siglo XVIII. Asclepio, v.53, n.1, p.141-171. 2001.

MOTTA, Pietro M.

Marcello Malpighi: a pioneer in embryology and reproduction. Progress in Clinical and Biological Research, v.296, p.3-6. 1989.

MRC...

MRC Vitamin Study Research Group. Prevention of neural tube defects: results of the Medical Research Council Vitamin Study. Lancet, v.338, n.8760, p.131-137. 1991.

NICHOL, David H.; WELCH, Arnold D. On the mechanism of action of aminopterin. Proceedings of the Society for Experimental Biology and Medicine, v.74, n.2, p.403-411. 1950.

NICHOLS, Buford. Introduction. [Symposium: Accomplishments in child nutrition during the 20th century]. The Journal of Nutrition, v.131, n.2, p.398S-400S. 2001. 
NZILA, Alexis et al.

Anticancer agents against malaria: time to revisit? Trends Parasitol, v.26, n.3, p.125-129. 2010.

OBLADEN, Michael.

Cats, frogs, and snakes: early concepts of neural tube defects. Journal of Child Neurology, v.26, n.11, p.1452-1461. 2011.

OLESON, Jacob et al.

Studies on the inhibitory nature of 4-aminopteroylglutamic acid. The Journal of Biological Chemistry, v.175, n.1, p.359-365. 1948.

PANGILINAN, Faith et al.

Evaluation of common genetic variants in 82 candidate genes as risk factors for neural tube defects. BMC Medical Genetics, v.13, n.62, p.62-81. 2012.

PARÉ, Ambroise.

Monstruos y prodigios. Madrid: Siruela. 1986.

PASTORE, Edward; FRIEDKIN, Morris.

The enzymatic synthesis of thymidylate. II. Transfer of tritium from tetrahydrofolate to the methyl group of thymidylate. The Journal of Biological Chemistry, v.237, n.12, p.3802-3810. 1962.

PIRO, Anna et al.

Casimir Funk: his discovery of the vitamins and their deficiency disorders. Annals of Nutrition and Metabolism, v.57, n.2, p.85-88. 2010.

REN, Aiguo et al.

Awareness and use of folic acid, and blood folate concentrations among pregnant women in northern China, an area with a high prevalence of neural tube defects. Reproductive Toxicology, v.22, n.3, p.431-436. 2006.

ROFAIL, Diana et al.

Factors contributing to the success of folic acid public health campaigns. Journal of Public Health, v.34, n.1, p.90-99. 2012.

ROSENBERG, Irwin H. et al.

Absorption of polyglutamic folate: participation of deconjugating enzymes of the intestinal mucosa. New England Journal of Medicine, v.280, n.18, p.985-988. 1969.

ROSENFELD, Louis.

Vitamine-vitamin. The early years of discovery. Clinical Chemistry, v.43, n.4, p.680-685. 1997.

SAINT-HILAIRE, Isidore Geoffroy.

Monstruosités, planche IX. In: Saint-Hilaire, Isidore Geoffreoy. Histoire générale et particulière des anomalies de l'organisation chez l'homme et les animaux. Paris: J.-B. Baillière. Disponible em: http://www.biusante.parisdescartes.fr/histmed/ medica/page?35541\&p=16. Acceso en: 23 mar. 2012. 1837.

SCHOLL, Theresa O.; JOHNSON, William G. Folic acid: influence on the outcome of pregnancy. American Journal of Clinical Nutrition, v.71, n.5, p.1295S-1303S. 2000.

SCOTT, John M.

Victor Herbert (1927-2002). Journal of Nutrition, v.134, n.7, p.1678-1680. 2004.

SEEGER, Doris et al.

Antagonist for pteroylglutamic acid. Journal of the American Chemical Society, v.69, n.10, p.2567. 1947.

SELLER, Mary J.

Sex, neural tube defects, and multisite closure of the human neural tube. American Journal of Medical Genetics, v.58, n.4, p.332-336. 1995.

SELLER, Mary J.

Neural tube defects and sex ratios. Lancet, v.2, n.8500, p.227. 1986.

SHANE, Barry; CARPENTER, Kenneth J. E.L. Robert Stokstad (1913-1995). Journal of Nutrition, v.127, n.2, p.199-201. 1997.

SIE, Karen et al.

Effect of maternal and post weaning folic acid supplementation on colorectal cancer risk in the offspring. Gut, v.60 n.12, p.1687-1694. 2011.

SMITHELLS, James R.W. et al.

Prevalence of neural tube defects in the Yorkshire Region. Community Medicine, v.11, n.2, p.163167. 1989.

SMITHELLS, James R.W. et al.

Neural-tube defects and vitamins: the need for a randomized clinical trial. British Journal of Obstetrics and Gynecology, v.92, n.2, p.185-188. 1985.

SMITHELLS, James R.W. et al.

Further experience of vitamin supplementation for prevention of neural tube defect recurrences. Lancet, v.321, n.8332, p.1027-1031. 1983.

SMITHELLS, James R.W. et al.

Possible prevention of neural-tube defects by periconceptional vitamin supplementation. Lancet, v.1, n.8164, p.339-340. 1980.

SMITHELLS, James R.W. et al.

Vitamin deficiencies and neural tube defects. Archives of Diseases in Childhood, v.51, n.12, p.944-950. 1976.

ST. CLAIR, John et al.

Methotrexate in abdominal pregnancy. JAMA, v.208, n.3, p.529-531. 1969. 
TABER, Larry A.

Towards a unified theory for morphomechanics. Philosophical Transactions of the Royal Society A, v.367, n.1902, p.3555-3583. 2009.

TEDSTONE, Alison et al.

Fortification of selected foodstuffs with folic acid in the UK: consumer research carried out to inform policy recommendations. Journal of Public Health, v.30, n.1, p.23-29. 2008.

THIERSCH, John B.

Therapeutic abortions with a folic acid antagonist, 4-aminopteroylglutamic acid (4-amino P.G.A) administered by the oral route. American Journal of Obstetrics and Gynecology, v.63, n.6, p.1298-1304. 1952.

TINKER, Sarah C. et al.

Does obesity modify the association of supplemental folic acid with folate status among nonpregnant women of childbearing age in the United States? Birth Defects Research Part A: Clinical and molecular Teratology, v.94, n.10, p.749-755. 2012.

TRUSSELL, James.

The cost of unintended pregnancy in the United States. Contraception, v.75, n.3, p.168-170. 2007.

VAN HEININGEN, T.W.

Maternal imagination and congenital malformations. Histoire des sciences médicales, v.45, n.3, p.239-248. 2011.

WALD, Nicholas J. et al.

Neural-tube defects and vitamins: the need for a randomized clinical trial. British Journal of Obstetrics and Gynaecology, v.91, n.6, p.516-523. 1984.

WALD, Nicholas J. et al.

Maternal serum-alpha-fetoprotein measurement in antenatal screening for anencephaly and spina bifida in early pregnancy. Report of U.K. collaborative study on alpha-fetoprotein in relation to neural-tube defects. Lancet, v.1, n.8026, p.1323-1332. 1977.
WANG, Hai-Gang et al.

Reduced folate carrier A80G polymorphism and susceptibility to neural tube defects: a metaanalysis. Gene, v.510, n.2, p.180-184. 2012.

WILLIAMS, Laura J. et al.

Prevalence of spina bifida and anencephaly during the transition to mandatory folic acid fortification in the United States. Teratology, v.66, n.1, p.33-39. 2002.

WILLS, Lucy.

Treatment of "pernicious anaemia of pregnancy" and "tropical anaemia" with special reference to yeast extract as a curative agent. 1931. Journal of Nutrition, v.7, n.5, p.323-327, discussion 328. 1991.

WILLS, Lucy; STEWART, Alice.

Experimental anaemia in monkeys, with special reference to macrocytic nutritional anaemia. British Journal of Experimental Pathology, v.16, n.5, p.444-453. 1935.

WOLF, George; CARPENTER, Kenneth J. Early research into the vitamins: the work of Wilhelm Stepp. Journal of Nutrition, v.127, n.7, p.1255-1259. 1997.

WOLFF, Tracy et al.

Folic acid supplementation for the prevention of neural tube defects: an update of the evidence for the U.S. Preventive Services Task Force. Annals of Internal Medicine, v.150, n.9, p.632-639. 2009.

ZANI, Augusto; COZZI, Denis A.

Giovanni Battista Morgagni and his contribution to pediatric surgery. Journal of Pediatric Surgery, v.43, n.4, p.729-733. 2008.

ZHANG, Ting et al.

Maternal serum vitamin B12, folate and homocysteine and the risk of neural tube defects in the offspring in a high-risk area of China. Public Health Nutrition, v.12, n.5, p.680-686. 2009.

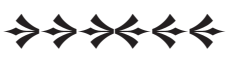

